2012-4

\title{
Analysis of a Kinetic Multi-Segment Foot Model. Part I: Model Repeatability and Kinematic Validity
}

\author{
Dustin A. Bruening \\ dabruening@byu.edu \\ Kevin M. Cooney \\ Frank L. Buczek
}

Follow this and additional works at: https://scholarsarchive.byu.edu/facpub

Part of the Exercise Science Commons

\section{Original Publication Citation}

Dustin A. Burening, Kevin M. Cooney, Frank L. Buczek. Analysis of a kinetic multi-segment foot model. Part I: Model repeatability and kinematic validity. Gait \& Posture. Volume 35, Issue 4, April 2012, Pages 529-534. doi:10.1016/j.gaitpost.2011.10.363

\section{BYU ScholarsArchive Citation}

Bruening, Dustin A.; Cooney, Kevin M.; and Buczek, Frank L., "Analysis of a Kinetic Multi-Segment Foot Model. Part I: Model Repeatability and Kinematic Validity" (2012). Faculty Publications. 1623.

https://scholarsarchive.byu.edu/facpub/1623 


\title{
Analysis of a kinetic multi-segment foot model. Part I: Model repeatability and kinematic validity
}

\author{
Dustin A. Bruening, Kevin M. Cooney, Frank L. Buczek
}

\begin{abstract}
Kinematic multi-segment foot models are still evolving, but have seen increased use in clinical and research settings. The addition of kinetics may increase knowledge of foot and ankle function as well as influence multi-segment foot model evolution; however, previous kinetic models are too complex for clinical use. In this study we present a three-segment kinetic foot model and thorough evaluation of model performance during normal gait. In this first of two companion papers, model reference frames and joint centers are analyzed for repeatability, joint translations are measured, segment rigidity characterized, and sample joint angles presented. Within-tester and between-tester repeatability were first assessed using 10 healthy pediatric participants, while kinematic parameters were subsequently measured on 17 additional healthy pediatric participants. Repeatability errors were generally low for all sagittal plane measures as well as transverse plane Hindfoot and Forefoot segments (median $<3^{\circ}$ ), while the least repeatable orientations were the Hindfoot coronal plane and Hallux transverse plane. Joint translations were generally less than $2 \mathrm{~mm}$ in any one direction, while segment rigidity analysis suggested rigid body behavior for the Shank and Hindfoot, with the Forefoot violating the rigid body assumptions in terminal stance/pre-swing. Joint excursions were consistent with previously published studies.
\end{abstract}

\section{Keywords}

Multi-segment foot; Foot models; Gait analysis; Kinetics; Repeatability

\section{Introduction}

The traditional single-segment foot model used in clinical gait analysis and human movement research is beginning to be replaced by various models in which the foot is divided into multiple rigid segments; however, evolution of these models is ongoing. Focus has shifted from equipment accuracy and resolution [1] and [2] to model repeatability [3], [4], [5] and [6], replication [5], [7] and [8], and clinical application [9], [10], [11], [12] and [13]. While kinematic 
analysis is now commonplace, the few models that have incorporated kinetics [14] and [15] have been hampered by excessive assumptions and equipment limitations [16], [17] and [18]. Anatomically meaningful reference frames that move with the segments of interest are sufficient for kinematics, whereas kinetic analysis additionally requires joint center definitions, segment inertial properties, ground reaction forces applied to separate segments of the foot, and a characterization of each segment's rigidity. Incorporation of these parameters may help increase knowledge of foot and ankle function as well as influence multi-segment foot model evolution. In this two-part study, we present a three-segment kinetic foot model and several analysis techniques used to characterize its performance during normal gait. Part I focuses on kinematics, including analyses of joint center and segment reference frame repeatability and characterizations of joint behavior and segment rigidity. Sample joint kinematics are also provided.

\section{Methods}

\section{Model}

The model consists of a Shank (tibia and fibula) and three foot segments: (1) Hindfoot (calcaneus and talus), (2) Forefoot (navicular, cuboid, cuneiforms, and metatarsals), and (3) Hallux (proximal and distal phalanges). While additional segments may be useful in future work (e.g. medial/lateral and/or midfoot/forefoot segmentation), they currently present hurdles in force measurement and repeatability [17] and [19].

In the current model (Table 1 and Table 2, Fig. 1), the Shank and Hindfoot are separated by the ankle complex (combined talocrural and subtalar joints), whose center (ACC) is defined by Bruening et al. [20]. The Hindfoot and Forefoot are separated by a mid-tarsal joint, with center (MTC) defined as the midpoint between markers placed over the navicular and cuboid bones. This is admittedly somewhat arbitrary, chosen for its easily palpable landmarks, but also conforms to the general finding that although motion exists among all bones of the midfoot, it is greatest proximally and medially [21] and [22]. The Forefoot and Hallux are separated by the 1st metatarsophalangeal joint (MP joint), whose center (MPC) is defined by a projection from a marker placed over the superior aspect of the 1st metatarsal head. Ankle and mid-tarsal joint motion were given six degrees-of-freedom (DOF), with Shank, Hindfoot, and Forefoot segments tracked independently [23], while the metatarsophalangeal (MP) joint was modeled with 2 DOF using inverse kinematics [24].

Table 1. Marker conventions and descriptions.

$\begin{array}{lll}\begin{array}{l}\text { Conv. } \\ \text { Markers }\end{array} & \text { Name } & \text { Description } \\ \text { LK } & \text { Lateral knee } & \text { Apex of lateral femoral epicondyle } \\ \text { MK } & \text { Medial knee } & \text { Apex of medial femoral epicondyle } \\ \text { S1-S4 } & \text { Shank shell } & \text { 4-Marker Shank cluster }\end{array}$




$\begin{array}{lll}\text { Conv. } & \text { Name } & \text { Description } \\ \text { LA } & \text { Lateral ankle } & \text { Apex of lateral malleolus } \\ \text { MA } & \text { Medial ankle } & \text { Apex of medial malleolus } \\ \text { C1 } & \text { Calcaneus 1 } & \text { Apex of calcaneal tuberosity } \\ \text { C2 } & \text { Calcaneus 2 } & \text { Superior apex of calcaneus (Achilles tendon insertion) } \\ \text { LC } & \text { Lateral calcaneus } & \text { Lateral calcaneus (peroneal tubercle) } \\ \text { MC } & \text { Medial calcaneus } & \text { Medial calcaneus (sustentaculum tali) } \\ \text { NV } & \text { Navicular } & \text { Medial prominence of navicular bone } \\ \text { CU } & \text { Cuboid } & \text { Lateral centroid of cuboid bone } \\ \text { B1 } & \text { Base 1 } & \text { Medial aspect of 1st metatarsal base } \\ \text { B5 } & \text { Base } 5 & \text { Lateral aspect of 5th metatarsal base } \\ \text { H2 } & \text { Head 2 } & \text { Midway between 2nd and 3rd metatarsal heads } \\ \text { HX } & \text { Hallux } & \text { Centroid of hallux nail } \\ \text { H1 } & \text { Head 1 } & \text { Superior aspect of 1st metatarsal head }\end{array}$

Virtual landmarks

$\begin{array}{lll}\text { KJC } & \text { Knee joint center } & \text { Midpoint (LK, MK) } \\ \text { ACC } & \text { Ankle complex center Midpoint (LA, MA), adjusted per Bruening et al. [20] } \\ \text { MTC } & \text { Mid-tarsal center } & \text { Midpoint (CU, NV) } \\ \text { MPC } & \text { 1st MP center } & \text { Projection H1 vertically 1/2 distance to floor } \\ \text { FF_Dist } & \text { Forefoot distal end } & \text { Projection H2 vertically 1/2 distance to floor } \\ \text { HX_Dist } & \text { Hallux distal end } & \text { Projection HX vertically 1/2 distance to floor }\end{array}$

Table 2. Segment reference frames, succinctly defined by a long axis (primary axis), a plane (the secondary axis is perpendicular to the plane, and tertiary axis perpendicular to primary and secondary axes), and the markers used to track the segment motion. All reference frames are oriented similar to the whole body planes in the static pose (mediolateral $(\mathrm{M} / \mathrm{L})$ axis laterally to subject's right side, anteroposterior (A/P) axis anterior, inferosuperior (Inf/Sup) axis upward).

$\begin{array}{llll}\text { Segment } & \text { Long axis } & \text { Plane } & \text { Tracking markers } \\ \text { Shank } & \text { KJC to ACC } & \text { KJC, LA, MA (coronal) } & \text { S1-S4 } \\ \text { Hindfoot } & \text { C1 to MTC } & \text { C1, MTC, C2 (sagittal) } & \text { C1, LC, MC } \\ \text { Forefoot } & \text { MTC to FF_Dist } & \text { MTC, FF_Dist, H2 (sagittal) } & \text { B1, B5, H2 } \\ \text { Hallux } & \text { MPC to HX_Dist } & \text { MPC, HX_Dist, H1 (sagittal) } & \text { HX, H1 } \\ \text { Foot }^{\mathrm{a}} & \text { ACC to FF_Dist } & \text { ACC, FF_Dist, H2 (sagittal) } & \text { C1, B1, B5, H2 }\end{array}$

a Foot segment used only in part II, but defined here for convenience. 


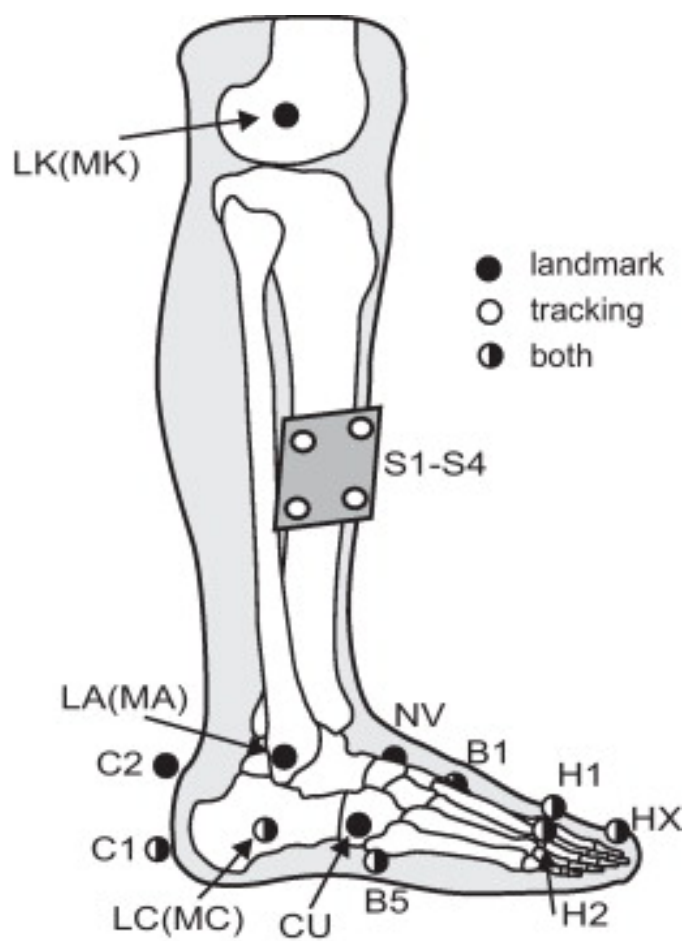

Fig. 1. Marker locations. The legend differentiates between markers that are used to define landmarks in the static pose, markers used only for tracking, and markers used for both. Hidden medial markers are noted on the figure in parentheses beside their lateral counterparts.

\section{Protocol overview}

Two studies were utilized with two separate but similar healthy pediatric participant samples. All participants were volunteers and signed consent/assent forms approved by the local human subjects committee. Pediatric participants were chosen because they are a specific target population for future model applications, comprise a range of foot sizes, and are more representative of marker placement challenges. The same equipment and software were used for both studies. A 10-camera Vicon 612 system (Vicon, Oxford England, UK) collecting at $120 \mathrm{~Hz}$ was used to track the positions of 19, 4-mm diameter spherical retro-reflective markers attached to the right foot and lower leg (Table 1, Fig. 1), and all data analysis was performed in Visual3D software (C-motion Inc., Rockville, MD, USA). Static trial marker trajectories were averaged over a small frame range, while walking marker trajectories were filtered using a low pass Butterworth filter (6 Hz cutoff frequency).

\section{Model repeatability protocol}


Model repeatability was assessed using 10 pediatric participants $(6 \mathrm{M} / 4 \mathrm{~F}$; age $13.7 \pm 3.5$; height $161 \pm 12 \mathrm{~cm}$; weight $54 \pm 15 \mathrm{~kg}$; mean \pm SD). Two researchers, each with several years of experience in marker placement and instrumented gait analysis, performed the single session tests as follows. The between-tester protocol began with the application of all markers by the first researcher. Non-tracking markers were covered with opaque tape (to reduce marker crowding), and three walking trials were collected. The tape was then removed and a static pose trial (SP1) was collected. The participant remained in place while the first researcher removed all of the markers and wiped off any markings that might guide the second researcher. Without the participant moving, the second researcher then placed all markers on the participant and a second static pose trial (SP2) was collected. The opaque tape was again applied to the non-tracking markers and three more walking trials were collected. The within-tester protocol then began by again removing the tape and collecting a third static pose trial (SP3). While the participant remained in place, the second researcher removed all markers and any markings that could guide the re-application of markers. The second researcher then re-applied all markers, and a fourth static pose trial (SP4) was collected. Non-tracking markers were removed and three final walking trials were collected, concluding the repeatability tests. Note that the knee markers and Shank cluster were left in place throughout all between and within-tester procedures, removing from consideration any variability their re-application could have added to the results.

Static pose trial repeatability was quantified by differences between-testers (SP1 and SP2) and within-testers (SP3 and SP4). Three variables were analyzed: (1) global joint center positions, (2) global segment orientation angles, and (3) relative joint angles. Segment orientation angles were calculated as the helical angles between segment and laboratory reference frames, chosen to avoid rotation order dependence. Joint angles were calculated using a Cardan rotation sequence between distal and proximal (reference) segments (1 - flexion/extension, 2 abduction/adduction, 3 - internal/external rotation).

Walking trials were calibrated from associated static poses, taken either after (SP1) or before (SP4) the walking trials. The second set of walking trials had two associated static poses (SP2 and SP3), so these trials were calibrated twice, with SP2 used for between-subjects comparisons and SP3 used for within-subjects comparisons. For all walking trials, joint angles were calculated using the same rotation sequence as above, then time normalized to percent gait cycle. Upon visual inspection of the data, there was good kinematic agreement among the three walking trials of each test, so these were ensemble averaged and the mean value (mean of the means) was used for comparisons.

\section{Kinematic validity protocol}

The term validity is used loosely here, but was chosen to encompass measures of joint translations and segment rigidity, as well as sample joint angles for comparison with previous studies. The kinematic validity study portion was performed on 17 participants (9M/8F; age $12.6 \pm 3.4$; height $154 \pm 18 \mathrm{~cm}$; weight $48 \pm 17 \mathrm{~kg}$; mean \pm SD). A separate participant sample was utilized so that the information gained from the repeatability protocol could be incorporated. A single researcher placed all markers, and additionally employed calipers and a graphic pencil 
to bisect the calcaneus and guide the placement of markers C1 and C2. For each participant, a static pose trial was collected, non-tracking markers removed, and three walking trials collected.

Joint translations were defined as the Euclidean distance between adjacent segment endpoints. Segment rigidity was assessed using the residual from the segment tracking algorithm [23], which is a measure of the goodness of fit of the tracking marker configuration at each walking frame compared to the static pose. For comparison purposes, the residual was normalized by the average inter-marker distance for each segment's tracking markers. Joint angles were again calculated using the above rotation sequence. All variables were time-normalized to percent gait cycle. A representative trial from each participant was chosen, and ensemble averages across participants were used for presentation.

\section{Results}

Differences in all repeatability variables are displayed as box-and-whiskers plots, chosen to show the full error distributions including absolute ranges (Fig. 2). Median differences in global joint center positions (Fig. 2A) were generally less than $2 \mathrm{~mm}$ in any one direction, with a high of $3.3 \mathrm{~mm}$ between-testers for the MTC in the A/P direction. The greatest difference for a single participant was $7.0 \mathrm{~mm}$ for the same joint in the same direction. Differences in A/P MTC location did not have an overly detrimental effect on segment orientations, as sagittal and transverse plane Hindfoot and Forefoot differences were relatively small. For all orientation and angle measurements (Fig. 2B-D), between-tester differences were generally greater than withintester differences, and an overall increase in differences was seen from static segment orientations to static joint angles to walking joint angles. Global segment orientation angles (Fig. 2B) showed median differences less than $3^{\circ}$ within-tester and less than $4^{\circ}$ between-testers, with the exception of the Hindfoot coronal plane angle $\left(5.3^{\circ}\right)$. This measure had a single participant high of $12.0^{\circ}$ between-testers, and affected both the frontal plane ankle (median $4.5^{\circ}$, high $10.8^{\circ}$ ) and mid-tarsal (median $5.9^{\circ}$, high $10.7^{\circ}$ ) static joint angles. These same angles were also the least repeatable in the walking trials (Fig. 2D), with differences up to $13.1^{\circ}$. The range of Hallux transverse plane orientation differences was also comparatively large, with a high of $9.8^{\circ}$ between-testers, carrying over to a high of $11.4^{\circ}$ between-testers in the static MP joint angles. 

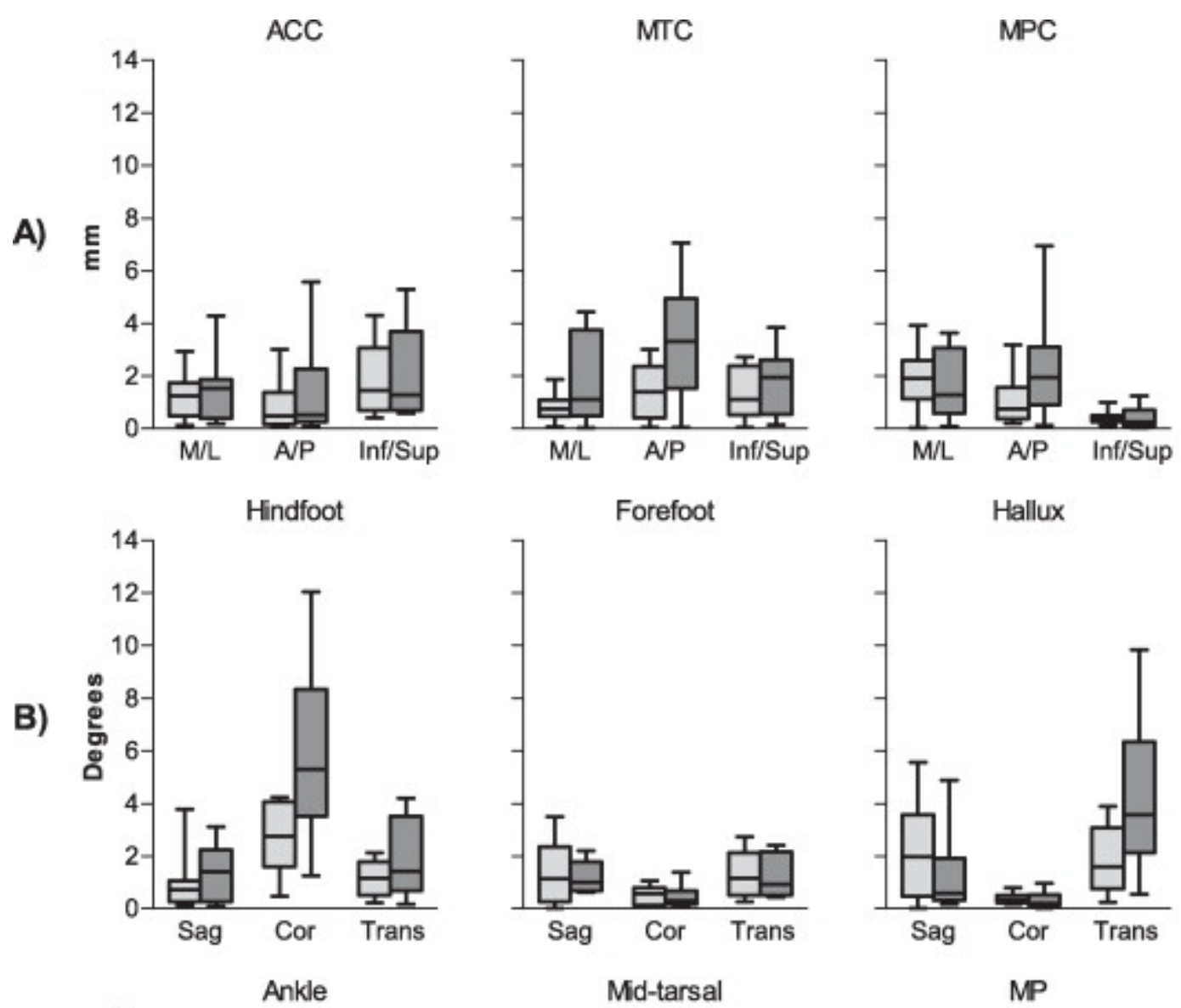

Forefoot

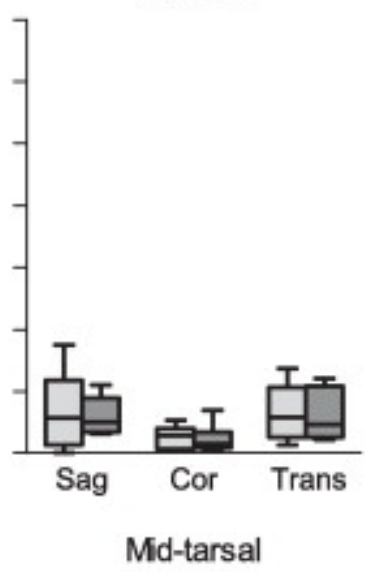

Hallux
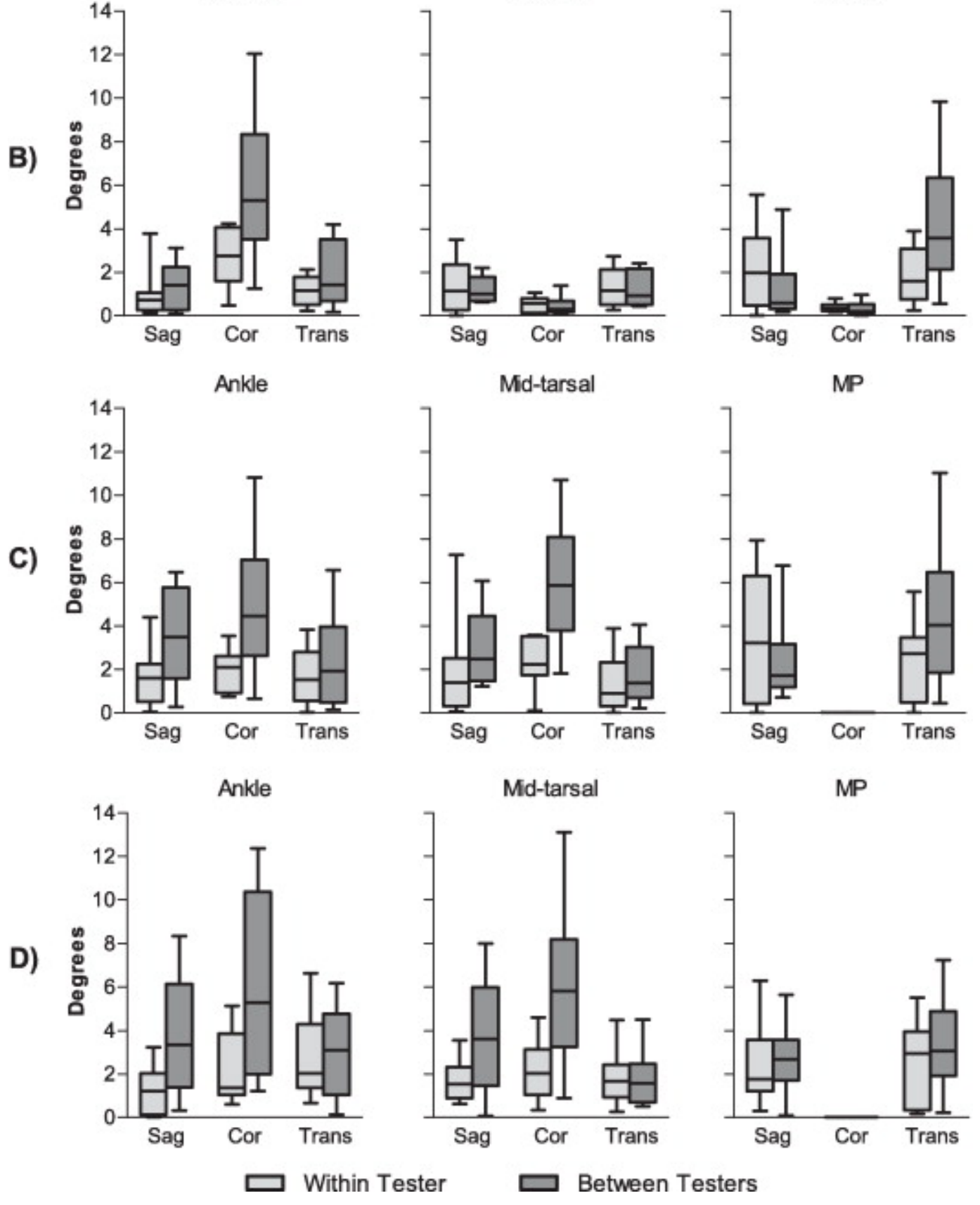
Fig. 2.

Within and between-tester differences displayed as box-and-whiskers plots (box includes median and 1st and 3rd quartiles, whiskers at max and min, $n=10$ subjects in the first experimental group): (A) static trial global joint center positions in the global M/L, A/P, and Inf/Sup directions; (B) static trial global segment orientation angles in the sagittal (Sag), coronal (Cor), and transverse (Trans) planes; (C) static trial joint angles; (D) walking trial mean joint angles.

Across trials and gait cycle, the mean joint translations (Fig. 3A) were $5.8 \mathrm{~mm}$ and $4.0 \mathrm{~mm}$ for the ankle and midfoot joints, respectively. All joint translations peaked in terminal stance/preswing, where the greatest deformation of the foot structures (compared to the static pose) occurred. Normalized Shank and Hindfoot segment residuals (Fig. 3B) had very little fluctuations across the gait cycle, while the Forefoot residuals increased twofold in pre-swing. Average inter-marker distances (used for normalization) were $7.7 \pm 0.6 \mathrm{~cm}$ for the Shank, $6.2 \pm 0.6$ for the Hindfoot, and $8.2 \pm 0.9 \mathrm{~cm}$ for the Forefoot. Joint angles showed relatively consistent patterns across participants (Fig. 4), and are comparable to previous studies [5], [8], [14] and [21].

A)
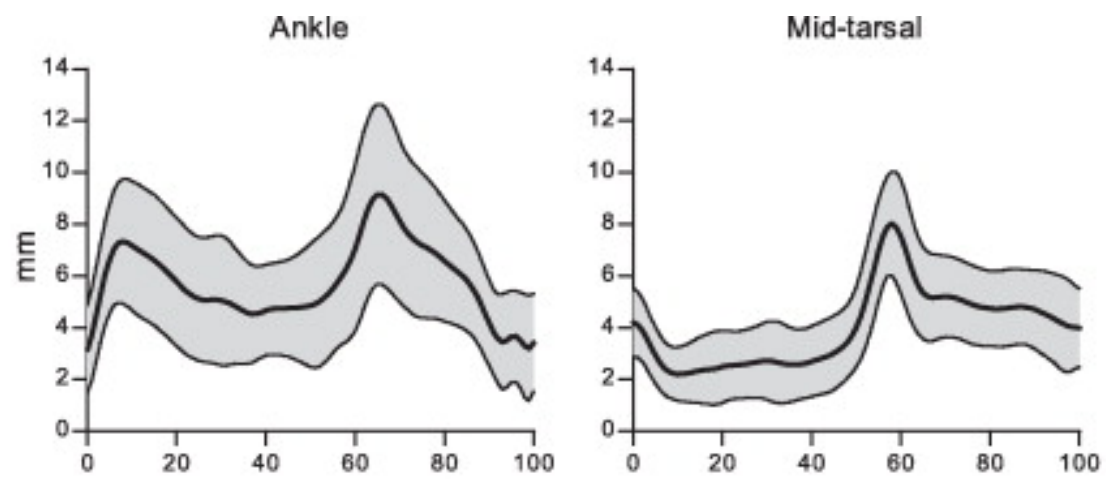

Shank
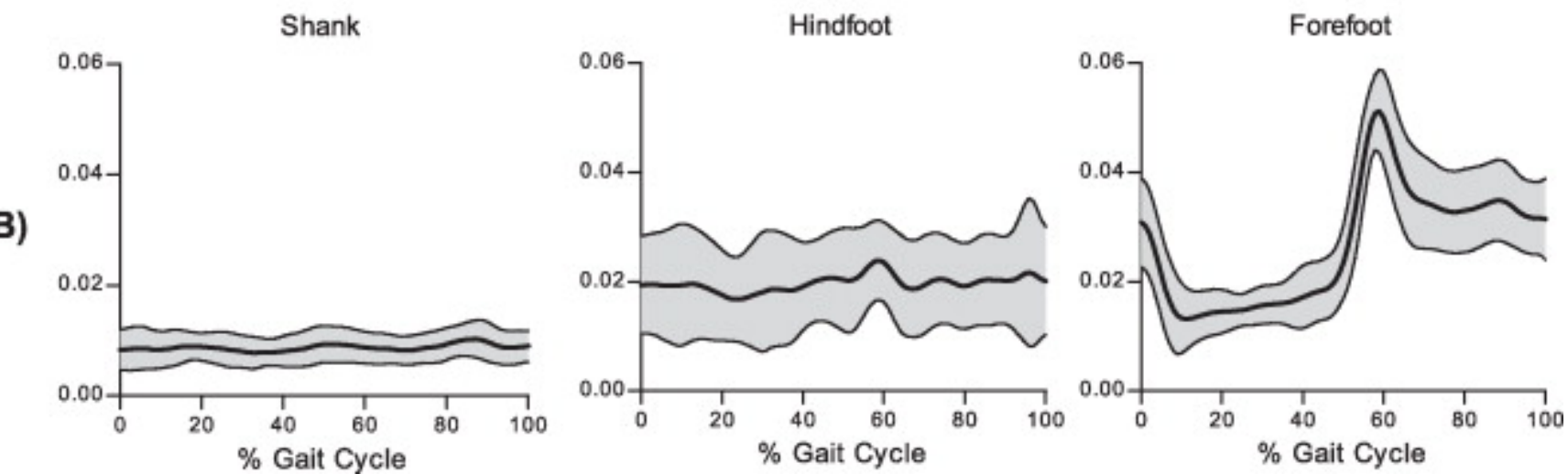

Fig. 3. 
Ensemble average joint translations (A), and normalized segment residuals (B) $(n=17$ subjects in the second experimental group). Note that the MP joint was modeled with 2 DOF (no translations). The segment residuals were normalized by average inter-marker distance.
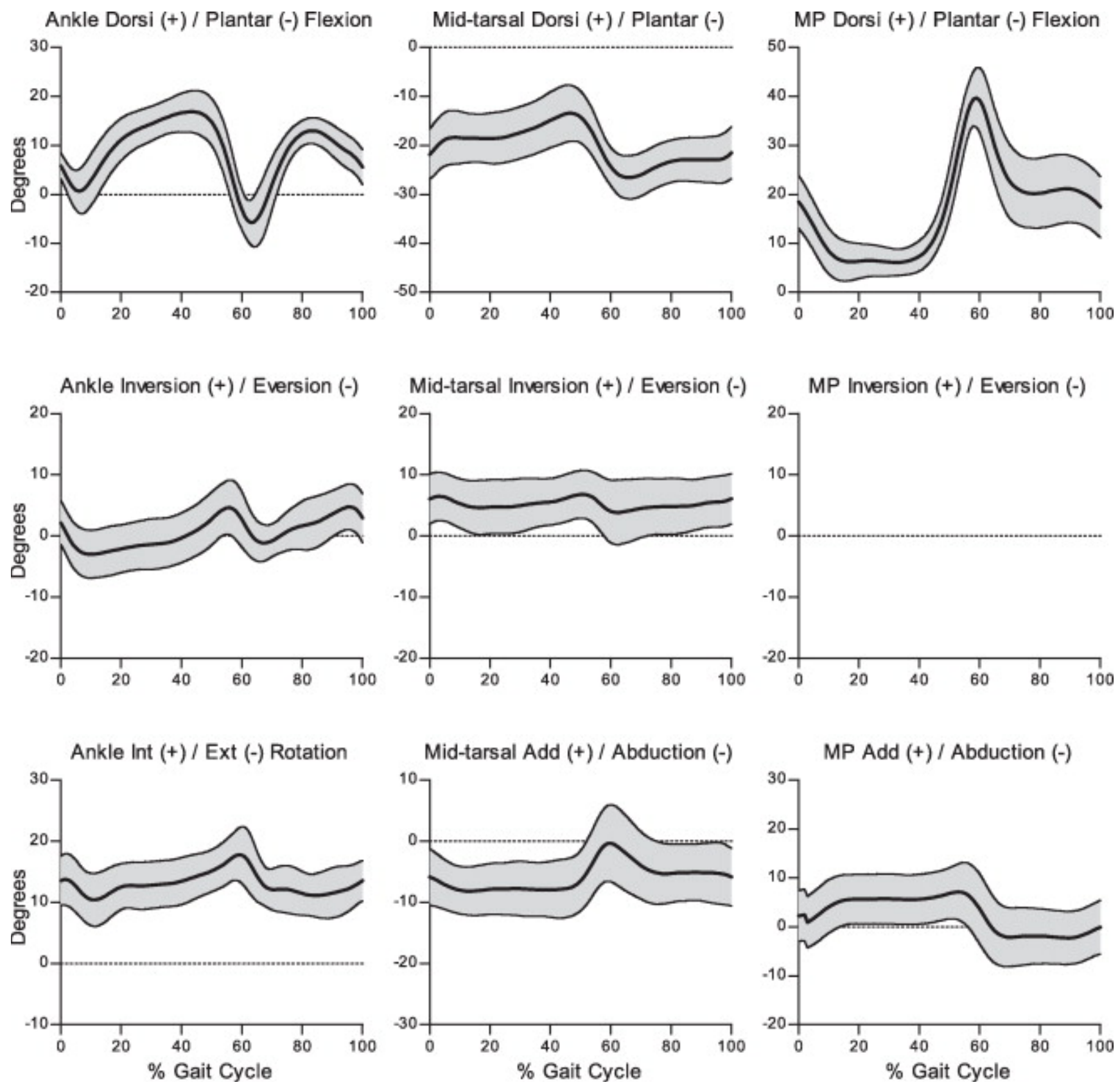

Fig. 4.

Ensemble average kinematics (joint rotation angles) during normal walking ( $n=17$ subjects in the second experimental group). The MP joint was modeled with 2 DOF (no coronal plane angles). 


\section{Discussion}

In this first of two companion papers we defined a three-segment kinetic foot model and used several techniques to analyze its kinematic performance. The model requires fewer assumptions regarding joint motion (e.g. ankle and mid-tarsal joints treated as 6 DOF) than previous kinetic models. Marker requirements are comparable to existing kinematic models, with the addition of two markers to define the MTC; however, these were needed only for the static pose. While there are several possible variations of landmark locations and kinematic segment definitions available, we relied primarily on joint centers and bony segment geometry to guide the model development, in preparation for kinetic application. This resulted in a few novel segment orientations; however, it should be stressed that while most software requires kinetic segments linked by joint centers, kinematic reference frames can co-exist in the same model for alternate representations of joint motion.

The Hindfoot segment orientation in this model was based solely upon bony anatomy, rather than the plantar surface [5] or some type of neutral positioning [2] and [4], which can be difficult for some patients to maintain. Defining the long axis using C1 and MTC results in a more dorsiflexed ankle position (and plantarflexed mid-tarsal position) than previous models. However, this axis is closely aligned with the radiographic calcaneal inclination angle [25]; an offset can also be used to adjust the baseline if desired. The limited fluctuations in the Hindfoot residual (Fig. 3B) suggest that this segment can be confidently treated as a rigid body with little detrimental soft tissue motion (even at C1 with interaction from the heel fat pad). Repeatability analysis showed that the least repeatable measure of the entire model was the Hindfoot coronal plane, where one participant had a difference of $12^{\circ}$ between-testers in static segment orientation (Fig. 2B). Difficulty in aligning this plane is due to subjective visual calcaneal bisection coupled with the small spacing between C1 and C2. Additionally, C2 is placed over the Achilles tendon insertion which has larger soft tissue motion, and slight variations in standing position between marker application and static pose collection (SP1 and SP3) may have slightly affected its position. LaPointe et al. [26] showed good calcaneal bisection repeatability when using calipers compared with visual alignment. For these reasons, calipers and a graphic pencil were used in the kinematic validity trials. There are alternate options to define frontal plane calcaneal orientation, but each has its own difficulties. Using the peroneal tubercle and sustentaculum tali in a slight varus offset has been suggested [8], but these landmarks can be difficult to palpate and also have limited spacing in pediatric feet. Using the ACC in place of C2 is another possibility [20], but the vertical distance between these two markers is potentially very small.

The Forefoot segment may have the most possible variations in definition, orientation, and tracking, as it encompasses several bones. For simplicity, we chose a Forefoot definition using the global reference frame to calibrate the distal endpoint and coronal plane orientation. Most patients are more able to position the distal end of the foot than the proximal; but certainly, other options are available. Forefoot segment tracking markers (B1, B5, H2) were chosen in an attempt to maximize segment rigidity. Still, the segment residual analysis suggested deformable behavior at the transitions between stance and swing, consistent with other studies [27]. The joints of the $\mathrm{mid} /$ forefoot are likely synergistic, though, and many valid conclusions can still be drawn. 
Alternate kinematic segments can also be included in addition to the kinetic model depending on the application (e.g. medial and lateral forefoot segments).

The small size of the Hallux presents some difficulties in marker spacing. For this reason we included both proximal and distal phalanges. Still, between-tester repeatability differences were increased in the transverse plane, apparently due to the A/P positioning of marker H1 (MPC differences). Alternate definitions are available (e.g. Stebbins et al. [5]), but were not tested. The MP joint was given just two DOF to reduce the number of tracking markers needed on the Hallux segment. This is a reasonable assumption based on the joint anatomy; however, the inverse kinematics algorithm relies on the coupled motion of the Hallux and Forefoot. Since the Forefoot exhibited non-rigid body behavior and is tracked by markers some distance from the joint itself, it may be possible to improve MP joint fidelity using an alternate kinematic segment tracked by medial markers (e.g. B1, H1, H2). Nevertheless, sagittal plane MP joint excursions are large, and the current model is likely sufficient for many applications.

Joint translations were used as one measure of joint center validity, particularly for the newly introduced MTC. The motion of the Forefoot relative to the Hindfoot of course encompasses several joints, each with multiple DOF including translations. Mid-tarsal motion is likely insufficient to accurately identify a functional joint using current methodology; thus the MTC definition used here is based on palpable landmarks which are reasonably repeatable (Fig. 2A). The relatively low mid-tarsal joint translations (i.e. lower than ankle, Fig. 3A) support the use of the proposed MTC definition, at least as an initial estimate. It should be noted that ankle translations were also greater than previously reported [20], likely due to the use of a shell mounted marker cluster on the Shank as opposed to a more longitudinally dispersed marker arrangement.

Although the segment reference frame orientations differ slightly from other kinematic models, the joint excursions (Fig. 4) closely match those previously reported [5], [8], [14] and [21]. Accounting for offsets, ankle and mid-tarsal curves very closely align with Stebbins et al. [5], who used similar tracking markers. The Hallux segment definition and MP joint angles are more comparable to Leardini et al. [8]. The Cardan rotation sequence used in this study is consistent with the majority of foot and lower extremity models, and, although this kinematic representation has proven useful, it is acknowledged that it does not fully address anatomical joint axes, which are rarely orthogonal, stationary, or consistent among participants or movements (particularly in the foot and ankle). Methods to determine joint axes and/or alternative kinematic representations should continue to be sought [28] and [29].

The repeatability analysis used in this study differs from previous studies, which have focused almost exclusively on walking trial variability [5], [6] and [30]. While between-day repeatability cannot be assessed using the current method, we were able to separate errors due to marker placement from variability inherent in gait, and isolate problematic markers and/or segment definitions. Our results contrast slightly with Stebbins et al. [5], who found the poorest repeatability in the transverse plane ankle and mid-tarsal joints. Stebbins visually aligned the transverse plane hindfoot orientation, while we used an anatomical landmark (MTC). This contrast, combined with the calcaneal bisection repeatability results previously mentioned, suggest that anatomical landmarks in general offer a repeatability advantage over visual 
alignment. Overall, the potential for substantial repeatability errors cannot be ignored, and highlights the need for consistent marker placement and clinician agreement.

The segment residual analysis was used as a rough indicator of rigidity. Residuals from the tracking algorithm arise due to soft tissue artifact, equipment noise, and rigid-body violations. Residual magnitudes are dependent upon marker spacing, and for this reason we have normalized the residuals by average inter-marker distance. While this is still not a perfect comparison method, it is a useful one, particularly when focusing on the changes in residual across the gait cycle. Contrasting residual fluctuations from the Shank shell (which is a true rigid body) with the Forefoot provides a good indicator of the relative effect of equipment noise vs. soft tissue artifact/rigid body violations.

As kinetic parameters and analysis are added in part II, and as the model is applied to clinical populations, additional insights into foot function and pathology may be possible. It is certainly important that both clinicians and researchers understand the repeatability tolerances, rigidity violations, and other limitations when designing studies around and interpreting results from multi-segment foot models.

\section{References}

[1] S.M. Kidder, F.S. Abuzzahab Jr., G.F. Harris, J.E. Johnson. A system for the analysis of foot and ankle kinematics during gait. IEEE Trans Rehabil Eng, 4 (1) (1996), pp. 25-32

[2] W. Liu, S. Siegler, H. Hillstrom, K. Whitney. Three-dimensional, six-degrees-offreedom kinematics of the human hindfoot during the stance phase of level walking. Hum Mov Sci, 16 (1997), pp. 283-298

[3] J. Henley, J. Richards, D. Hudson, C. Church, S. Coleman, L. Kerstetter, et al. Reliability of a clinically practical multi-segment foot marker set/model. G.F. Harris, P.A. Smith, R.M. Marks (Eds.), Foot and ankle motion analysis_clinical treatment and technology, CRC Press Taylor and Francis Group, LLC, Boca Raton (2008), pp. 445-464

[4] L. Moseley, R. Smith, A. Hunt, R. Gant. Three-dimensional kinematics of the rearfoot during the stance phase of walking in normal young adult males. Clin Biomech (Bristol, Avon), 11 (1) (1996), pp. 39-45

[5] J. Stebbins, M. Harrington, N. Thompson, A. Zavatsky, T. Theologis. Repeatability of a model for measuring multi-segment foot kinematics in children. Gait Posture, 23 (4) (2006), pp. 401-410 
[6] D.J. Curtis, J. Bencke, J.A. Stebbins, B. Stansfield. Intra-rater repeatability of the Oxford foot model in healthy children in different stages of the foot roll over process during gait. Gait Posture, 30 (1) (2009), pp. 118-121

[7] M.C. Carson, M.E. Harrington, N. Thompson, J.J. O’Connor, T.N. Theologis. ematic analysis of a multi-segment foot model for research and clinical applications: a repeatability analysis. J Biomech, 34 (10) (2001), pp. 1299-1307

[8] A. Leardini, M.G. Benedetti, L. Berti, D. Bettinelli, R. Nativo, S. Giannini. Rearfoot, mid-foot and fore-foot motion during the stance phase of gait. Gait Posture, 25 (3) (2007), pp. 453-462

[9] M.E. Ness, J. Long, R. Marks, G. Harris. Foot and ankle kinematics in patients with posterior tibial tendon dysfunction. Gait Posture, 27 (2) (2008), pp. 331-339

[10] T.N. Theologis, M.E. Harrington, N. Thompson, M.K. Benson. Dynamic foot movement in children treated for congenital talipes equinovarus. J Bone Joint Surg Br, 85 (4) (2003), pp. 572-577

[11] J. Tome, D.A. Nawoczenski, A. Flemister, J. Houck. Comparison of foot kinematics between subjects with posterior tibialis tendon dysfunction and healthy controls. J Orthop Sports Phys Ther, 36 (9) (2006), pp. 635-644

[12] D.E. Turner, P.S. Helliwell, K.L. Siegel, J. Woodburn. Biomechanics of the foot in rheumatoid arthritis: identifying abnormal function and the factors associated with localised disease 'impact’. Clin Biomech (Bristol, Avon), 23 (1) (2008), pp. 93-100

[13] W.L. Wu, F.C. Su, Y.M. Cheng, P.J. Huang, Y.L. Chou, C.K. Chou. Gait analysis after ankle arthrodesis. Gait Posture, 11 (1) (2000), pp. 54-61

[14] B.A. MacWilliams, M. Cowley, D.E. Nicholson. Foot kinematics and kinetics during adolescent gait. Gait Posture, 17 (3) (2003), pp. 214-224

[15] S.H. Scott, D.A. Winter. Biomechanical model of the human foot: kinematics and kinetics during the stance phase of walking. J Biomech, 26 (9) (1993), pp. 10911104

[16] D.A. Bruening, K.M. Cooney, F.L. Buczek, J.G. Richards. Measured and estimated ground reaction forces for multi-segment foot models. J Biomech, 43 (16) (2010), pp. 3222-3226

[17] F.L. Buczek, M.R. Walker, M.J. Rainbow, K.M. Cooney, J.O. Sanders. Impact of mediolateral segmentation on a multi-segment foot model. Gait Posture, 23 (4) (2006), pp. 519-522 
[18] M. Yavuz, G. Botek, B.L. Davis. Plantar shear stress distributions: comparing actual and predicted frictional forces at the foot-ground interface. J Biomech, 40 (13) (2007), pp. 3045-3049

[19] N. Wrbaskic, J.J. Dowling. An investigation into the deformable characteristics of the human foot using fluoroscopic imaging. Clin Biomech (Bristol, Avon), 22 (2) (2007), pp. 230-238

[20] D.A. Bruening, A.N. Crewe, F.L. Buczek. A simple, anatomically based correction to the conventional ankle joint center. Clin Biomech (Bristol, Avon), 23 (10) (2008), pp. 1299-1302

[21] P. Lundgren, C. Nester, A. Liu, A. Arndt, R. Jones, A. Stacoff, et al. Invasive in vivo measurement of rear-, mid- and forefoot motion during walking. Gait Posture, 28 (1) (2008), pp. 93-100

[22] C. Nester, R.K. Jones, A. Liu, D. Howard, A. Lundberg, A. Arndt, et al. Foot kinematics during walking measured using bone and surface mounted markers. J Biomech, 40 (15) (2007), pp. 3412-3423

[23] C.W. Spoor, F.E. Veldpaus. Rigid body motion calculated from spatial coordinates of markers. J Biomech, 13 (4) (1980), pp. 391-393

[24] T.W. Lu, J.J. O’Connor. Bone position estimation from skin marker co-ordinates using global optimisation with joint constraints. J Biomech, 32 (2) (1999), pp. 129_ 134

[25] M. Razeghi, M.E. Batt. Foot type classification: a critical review of current methods. Gait Posture, 15 (3) (2002), pp. 282-291

[26] S.J. LaPointe, C. Peebles, A. Nakra, H. Hillstrom. The reliability of clinical and caliper-based calcaneal bisection measurements. J Am Podiatr Med Assoc, 91 (3) (2001), pp. 121-126

[27] N. Okita, S.A. Meyers, J.H. Challis, N.A. Sharkey. An objective evaluation of a segmented foot model. Gait Posture, 30 (1) (2009), pp. 27-34

[28] G.S. Lewis, H.J. Sommer 3rd, S.J. Piazza. In vitro assessment of a motion-based optimization method for locating the talocrural and subtalar joint axes. J Biomech Eng, 128 (4) (2006), pp. 596-603

[29] J. Simon, L. Doederlein, A.S. McIntosh, D. Metaxiotis, H.G. Bock, S.I. Wolf. The Heidelberg foot measurement method: development, description and assessment. Gait Posture, 23 (4) (2006), pp. 411-424 
[30] J.L. McGinley, R. Baker, R. Wolfe, M.E. Morris. The reliability of threedimensional kinematic gait measurements: a systematic review. Gait Posture, 29 (3) (2009), pp. 360-369

NIOSH disclaimer: The findings and conclusions in this report are those of the authors and do not necessarily represent the views of the National Institute for Occupational Safety and Health. 